Research Article

\title{
Relationship between CRP Albumin Ratio and the Mortality in Critically Ill Patients with AKI: A Retrospective Observational Study
}

\author{
Jiajia Wang $\mathbb{D}^{1},{ }^{1}$ Kai Zhao $\mathbb{D}{ }^{2}$ Xuehui Mao $\mathbb{D},{ }^{3}$ Yabin Zhang $\mathbb{D},{ }^{4}$ Jing Shao $\mathbb{D},{ }^{4}$ \\ Weihua Fan $\left(\mathbb{D},{ }^{4}\right.$ and Yong Wang $\mathbb{D}^{4}$ \\ ${ }^{1}$ Department of Clinical Laboratory, Shandong Provincial Hospital, Cheeloo College of Medicine, Shandong University, Jinan, \\ Shandong 250021, China \\ ${ }^{2}$ Information Network Management Office, Shandong Provincial Hospital Affiliated to Shandong First Medical University, Jinan, \\ Shandong 250021, China \\ ${ }^{3}$ Department of Gastrointestinal Surgery, Shandong Provincial Hospital Affiliated to Shandong First Medical University, Jinan, \\ Shandong 250021, China \\ ${ }^{4}$ Department of Clinical Laboratory, Shandong Provincial Hospital Affiliated to Shandong First Medical University, Jinan, \\ Shandong 250021, China
}

Correspondence should be addressed to Yong Wang; slyywangyong@163.com

Received 23 March 2021; Accepted 11 September 2021; Published 30 September 2021

Academic Editor: Heng Jung Hsu

Copyright ( 2021 Jiajia Wang et al. This is an open access article distributed under the Creative Commons Attribution License, which permits unrestricted use, distribution, and reproduction in any medium, provided the original work is properly cited.

\begin{abstract}
Background. AKI is known to be associated with inflammation and nutritional status. The novel inflammatory prognostic score CAR (CRP/albumin ratio), which combines inflammation and nutritional status, was hypothesized to be associated with mortality in critically ill AKI patients in this study. Methods. The included cases were patients admitted to the ICU of Shandong Provincial Hospital from January 2016 to November 2018 and diagnosed with AKI within 48 hours of ICU admission. From the electronic case database of Shandong Provincial Hospital, we extracted the baseline demographic information, vital signs, routine laboratory parameters, complications, and other data. The above records are measured within 48 hours of admission to ICU. The clinical endpoint was the total cause mortality rate in hospital and 2 years. We constructed two multivariate regression models to determine the statistically significant correlation between CAR and mortality and conducted subgroup analysis to determine the mortality among different subgroups. Results. A total of 580 patients were included in this study. In multivariate regression analysis, higher CAR was associated with an increase in hospital and two-year all-cause mortality in critically ill patients with AKI after adjusting gender, age, respiratory frequency, temperature, and other confounding factors (tertile 3 versus tertile 1: OR, 95\% CI: 2.97, 1.70-5.17; 3.03, 1.68-5.47, respectively; $P<0.001$ ). Subgroup analysis showed that the CAR level in each subgroup increases with hospital mortality in critically ill patients with AKI. Conclusion. The increase of CAR in critically ill patients with AKI was associated with an increased risk of all-cause death.
\end{abstract}

\section{Introduction}

Acute kidney injury (AKI) is a severe clinical syndrome characterized by a rapid decline in renal function due to multiple causes with a higher incidence of morbidity and mortality, especially in patients admitted to intensive care units (ICU) $[1,2]$. The incidence of AKI in patients admitted to ICU is reported to be $36 \%$, with a higher mortality rate of $60-70 \%[3,4]$. Moreover, surviving patients often fail to recover renal function and require lifelong renal replacement therapy $[5,6]$, severely reducing their quality of life and increasing the financial burden. Given the high incidence and mortality of AKI, researchers actively investigate the various factors associated with AKI's occurrence and 
prognosis to take effective interventional measures to improve survival in the early stage. They have also made some achievements [7-9].

AKI is a complex disease with multiple etiologies and risk factors, usually following other acute and chronic conditions. Common causes of AKI include systemic inflammatory process, decreased kidney perfusion, obstruction of the urinary tract, and renal toxicity [10]. Among them, inflammation plays a key role. C-reactive protein (CRP), an essential major acute-phase protein, is the most commonly clinically used to reflect inflammation because the release of inflammatory cytokines causes its increased concentration and is associated with AKI mortality [11]. Albumin (ALB), the most abundant protein in plasma, is an indicator of human nutritional status and has also been reported to be associated with AKI [12]. Moreover, it has been proved that decreased ALB is associated with an inflammatory response [13]. Previous studies have demonstrated that higher CRP or lower ALB levels suggest a higher incidence of contrast agent-induced nephropathy $[12,14]$. Furthermore, as a novel inflammatory prognostic score, CRP/albumin ratio (CAR) is helpful in predicting inflammatory diseases and cancers [15-18]. Therefore, we hypothesized that a CAR score could use to assess AKI mortality.

\section{Methods}

2.1. Population Selection Criteria. This is a single-center retrospective study. The included cases were patients admitted to the ICU of Shandong Provincial Hospital from January 2016 to November 2018 and diagnosed with AKI within 48 hours of ICU admission. Basal creatinine value was the lowest value measured after this admission. The AKI was determined by kidney disease's definition: Improved Global Outcomes (KDIGO) guidelines [19]. Patients meeting the following criteria were excluded: (1) c-reactive protein or albumin was not detected during ICU stay, (2) first-time ICU admission was less than 24 hours, (3) age was less than 18 years, and (4) missing value $>5 \%$.

2.2. Data Extraction. Baseline demographic information, vital signs, routine laboratory parameters, complications, and other data were extracted from Shandong Provincial Hospital's electronic medical record system. Patient baseline demographic information and vital signs, including age, sex, mean arterial pressure (MAP), respiratory rate, heart rate, and temperature, were extracted. Complications include hypertension, coronary artery disease (CAD), kidney disease, and cancer. Routine laboratory parameters include CRP, ALB, urea nitrogen (BUN), creatinine, sodium, potassium, chloride, red blood cells (RBC), white blood cells (WBC), platelets, glucose, hematocrit, hemoglobin, mean corpuscular volume (MCV), triglycerides, total bilirubin (TBIL), red cell distribution width (RDW), and $\beta 2$-microglobulin (BMG). The above records are measured within 48 hours of admission to ICU. Other extracted data included the AKI stage, AKI recovery, surgical grade, length of stay in the ICU, and whether the death occurred in the hospital. The state of renal function recovery was determined accord-

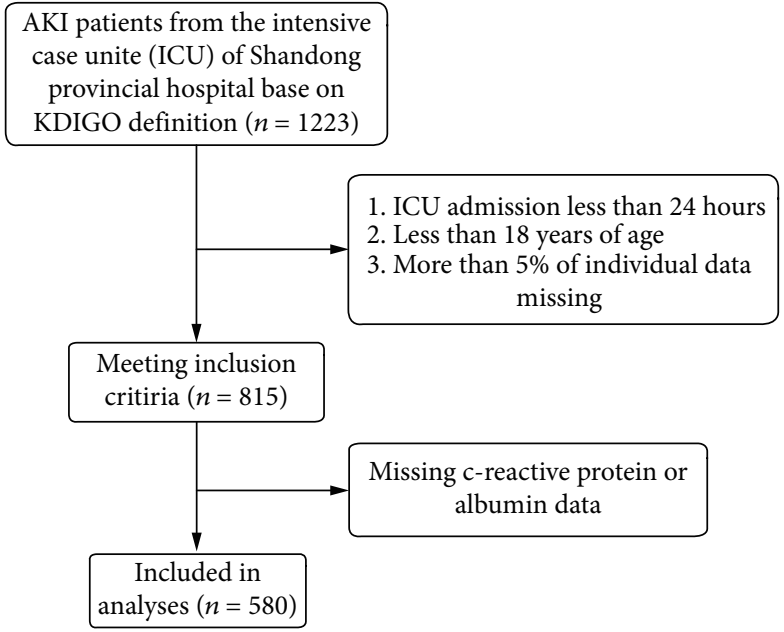

FIgURE 1: Illustration of exclusion criteria as utilized to select the final 580 patients.

ing to the last creatinine value before discharge. The last creatinine value $\leq 125 \%$ of base creatinine value was defined as recovery from AKI; otherwise, it is not recovered [20]. Two years after discharge, the included patients were followed up by telephone interviews to determine the twoyear survival rate. The end of the observation period was November 2020, or loss to follow-up. Sequential organ failure assessment (SOFA) score [21] and acute physiological and chronic health score II (APACHE II) [22] were also performed for each patient.

2.3. Statistical Analysis. CAR tertiles stratified the clinical characteristics of all included cases. Continuous variables are presented as means \pm SD or median (25th-75th percentile), and categorical data are described as numbers and percentages. The difference between the model development CAR tertiles groups was compared using Chi-squared tests for categorical variables and one-way analysis of variance (ANOVA) for continuous data. The variables with a skewed distribution used the Kruskal-Wallis test to compare. We performed multiple logistic regression analyses to determine the association between hospital mortality and 2-year mortality in CAR and AKI patients. We represented the odds ratio (OR) and 95\% confidence interval (CI). We construct two multiple regression models to identify a statistical significant correlation between CAR and mortality; CAR's first tertile was used as the reference group. These confounding factors were selected based on a change in influence estimated at over $10 \%$ or the $P$ values of regression coefficients $<0.1$ [23]. In model I, gender and age were adjusted as covariates. We adjusted for gender, age, respiratory rate, temperature, CAD, glucose, triglycerides, and surgical grade in model II. We performed a stratified analysis to assess whether the effects of CAR were consistent across subgroups classified by respiratory rate, temperature, hypertension, CAD, BUN, creatinine, sodium, potassium, WBC, RBC, glucose, hematocrit, hemoglobin, MCV, triglycerides, TBIL, RDW, BMG, recover from AKI or not, length of ICU stay, and surgical grade. 
TABLE 1: Characteristics of the study patients according to CAR.

\begin{tabular}{|c|c|c|c|c|}
\hline Characteristics & $0-0.2(n=193)$ & $\begin{array}{l}\text { CRP to albumin ratio } \\
0.21-2.53(n=191)\end{array}$ & $2.54-16.43(n=196)$ & $P$ value \\
\hline Age (years) & $57.3 \pm 14.9$ & $56.1 \pm 17.5$ & $62.6 \pm 15.1$ & $<0.001$ \\
\hline Gender $(n(\%))$ & & & & 0.022 \\
\hline Male & $111(57.5 \%)$ & $121(63.4 \%)$ & $139(70.9 \%)$ & \\
\hline Female & $82(42.5 \%)$ & $70(36.6 \%)$ & $57(29.1 \%)$ & \\
\hline CAR & $0.0(0.0-0.1)$ & $0.9(0.4-1.7)$ & $5.1(3.7-7.6)$ & $<0.001$ \\
\hline Respiratory rate (beats/minute) & $20.3 \pm 7.6$ & $20.9 \pm 7.2$ & $21.5 \pm 8.0$ & $<0.001$ \\
\hline Temperature $\left({ }^{\circ} \mathrm{C}\right)$ & $36.6 \pm 0.6$ & $36.6 \pm 0.7$ & $36.7 \pm 0.8$ & 0.036 \\
\hline Heart rate (beats/minute) & $91.2 \pm 20.6$ & $95.4 \pm 25.4$ & $96.5 \pm 26.5$ & 0.266 \\
\hline MAP (mmHg) & $96.8 \pm 21.3$ & $92.4 \pm 19.6$ & $92.9 \pm 21.8$ & 0.08 \\
\hline \multicolumn{5}{|l|}{ Laboratory parameters } \\
\hline Albumin $(\mathrm{g} / \mathrm{L})$ & $36.1 \pm 6.7$ & $32.9 \pm 7.4$ & $28.4 \pm 6.6$ & $<0.001$ \\
\hline CRP (mg/L) & $1.5(0.7-2.9)$ & $5.9(12.5-54.7)$ & $151.4(110.5-205.0)$ & $<0.001$ \\
\hline $\mathrm{BUN}(\mathrm{mmol} / \mathrm{L})$ & $6.1(4.6-9.0)$ & $7.6(5.2-12.6)$ & $8.0(5.3-12.5)$ & $<0.001$ \\
\hline Chloride (mmol/L) & $105.3 \pm 4.9$ & $105.4 \pm 33.8$ & $103.1 \pm 10.9$ & 0.005 \\
\hline Creatinine $(\mu \mathrm{mol} / \mathrm{L})$ & $154.4(118.0-211.9)$ & $179.5(124.4-254.8)$ & $182.8(131.0-252.0)$ & 0.029 \\
\hline Glucose $(\mathrm{mmol} / \mathrm{L})$ & $5.4(4.9-7.0)$ & $6.4(5.2-8.5)$ & $7.0(5.3-10.0)$ & $<0.001$ \\
\hline Potassium $(\mathrm{mmol} / \mathrm{L})$ & $4.3 \pm 0.6$ & $4.1 \pm 0.6$ & $4.1 \pm 0.7$ & 0.017 \\
\hline Sodium $(\mathrm{mmol} / \mathrm{L})$ & $140.1 \pm 3.4$ & $138.4 \pm 5.7$ & $139.1 \pm 11.8$ & 0.001 \\
\hline Triglycerides $(\mathrm{mmol} / \mathrm{L})$ & $1.2(0.9-1.8)$ & $1.3(0.9-1.9)$ & $1.4(0.9-2.1)$ & 0.151 \\
\hline $\mathrm{BMG}(\mathrm{mg} / \mathrm{L})$ & $2.5(1.9-3.5)$ & $3.0(2.2-5.1)$ & $3.0(2.2-5.6)$ & $<0.001$ \\
\hline Hematocrit (\%) & $37.6 \pm 6.8$ & $34.8 \pm 8.2$ & $35.4 \pm 8.4$ & 0.002 \\
\hline Hemoglobin $(\mathrm{g} / \mathrm{L})$ & $126.1 \pm 25.1$ & $115.4 \pm 28.8$ & $117.8 \pm 29.1$ & $<0.001$ \\
\hline $\mathrm{MCV}(\mathrm{fL})$ & $90.3 \pm 8.1$ & $89.3 \pm 9.1$ & $90.6 \pm 10.6$ & 0.017 \\
\hline Platelets $\left(10^{9} / \mathrm{L}\right)$ & $176.0(135.0-227.0)$ & $187.0(116.0-232.0)$ & $181.0(103.5-248.0)$ & 0.896 \\
\hline $\mathrm{RBC}\left(10^{12} / \mathrm{L}\right)$ & $4.2 \pm 0.8$ & $3.9 \pm 0.9$ & $3.9 \pm 0.9$ & 0.002 \\
\hline RDW (\%) & $13.8 \pm 1.8$ & $14.3 \pm 1.9$ & $14.3 \pm 2.4$ & 0.005 \\
\hline WBC $\left(10^{9} / \mathrm{L}\right)$ & $6.4(5.1-8.9)$ & $8.9(5.9-12.2)$ & $9.5(6.5-13.9)$ & $<0.001$ \\
\hline TBIL $(\mu \mathrm{mol} / \mathrm{L})$ & $13.9(10.2-19.7)$ & $15.2(10.8-22.5)$ & $15.9(10.9-26.5)$ & 0.047 \\
\hline \multicolumn{5}{|l|}{ Scoring systems } \\
\hline APACHE II & $12.0(8.0-17.0)$ & $15.0(10.0-20.0)$ & $18.0(12.0-23.0)$ & $<0.001$ \\
\hline SOFA & $4.0(2.0-5.0)$ & $5.0(3.0-7.5)$ & $5.0(3.0-8.0)$ & $<0.001$ \\
\hline \multicolumn{5}{|l|}{ Comorbidities ( $n(\%))$} \\
\hline Hypertension & $93(48.2 \%)$ & $73(38.2 \%)$ & $111(56.6 \%)$ & 0.001 \\
\hline Diabetes & $43(22.3 \%)$ & $32(16.8 \%)$ & $37(18.9 \%)$ & 0.383 \\
\hline Coronary artery disease & $23(11.9 \%)$ & $16(8.4 \%)$ & $37(18.9 \%)$ & 0.008 \\
\hline Acute cerebral infarction & $26(13.5 \%)$ & $15(7.9 \%)$ & $27(13.8 \%)$ & 0.127 \\
\hline Cancer & $3(1.6 \%)$ & $4(2.1 \%)$ & $13(6.6 \%)$ & 0.011 \\
\hline Surgical grade $(n(\%))$ & & & & $<0.001$ \\
\hline No surgery or grade 1 & $51(26.4 \%)$ & $81(42.4 \%)$ & $66(33.7 \%)$ & \\
\hline Grade 2 & $44(22.8 \%)$ & $17(8.9 \%)$ & $15(7.7 \%)$ & \\
\hline Grade 3 & $15(7.8 \%)$ & $11(5.8 \%)$ & $10(5.1 \%)$ & \\
\hline Grade 4 & $83(43.0 \%)$ & $82(42.9 \%)$ & $105(53.6 \%)$ & \\
\hline AKI stage $(n(\%))$ & & & & 0.037 \\
\hline Stage 1 & $138(71.5 \%)$ & $115(60.2 \%)$ & $115(58.7 \%)$ & \\
\hline Stage 2 & $33(17.1 \%)$ & $55(28.8 \%)$ & $56(28.6 \%)$ & \\
\hline Stage 3 & $22(11.4 \%)$ & $21(11.0 \%)$ & $25(12.8 \%)$ & \\
\hline
\end{tabular}


TABLE 1: Continued.

\begin{tabular}{|c|c|c|c|c|}
\hline Characteristics & $0-0.2(n=193)$ & $\begin{array}{c}\text { CRP to albumin ratio } \\
0.21-2.53(n=191)\end{array}$ & $2.54-16.43(n=196)$ & $P$ value \\
\hline ICU LOS (days) & $4.0(2.0-9.0)$ & $7.0(3.5-12.0)$ & $9.0(4.0-15.0)$ & $<0.001$ \\
\hline Recovery from AKI $(n(\%))$ & $141(73.1 \%)$ & $129(66.8 \%)$ & $106(54.6 \%)$ & $<0.001$ \\
\hline Hospital mortality $(n(\%))$ & $28(14.5 \%)$ & $43(22.5 \%)$ & $75(38.3 \%)$ & $<0.001$ \\
\hline 2-year mortality $(n(\%))$ & $46(36.2 \%)$ & $69(57.5 \%)$ & $101(69.7 \%)$ & $<0.001$ \\
\hline
\end{tabular}

CAR: C-reactive protein-to-albumin ratio; CRP: C-reactive protein; MAP: mean arterial pressure; BUN: blood urea nitrogen; BMG: $\beta 2$-microglobulin; MCV: mean corpuscular volume; RBC: red blood cells; RDW: red cell distribution width; WBC: white blood cells; TBIL: total bilirubin; SOFA: sequential organ failure assessment; APACHE II: acute physiological and chronic health score II; AKI: acute kidney injury; ICU: intensive care unit; LOS: length of stay.

TABLE 2: Relationship between CAR and mortality.

\begin{tabular}{|c|c|c|c|c|c|c|}
\hline \multirow{2}{*}{ CAR } & \multicolumn{2}{|c|}{ Nonadjusted } & \multicolumn{2}{|c|}{ Model I } & \multicolumn{2}{|c|}{ Model II } \\
\hline & OR (95\% CIs) & $P$ value & OR (95\% CIs) & $P$ value & OR (95\% CIs) & $P$ value \\
\hline \multicolumn{7}{|l|}{ Hospital mortality } \\
\hline Per 1 sd change & $1.71(1.42,2.04)$ & $<0.0001$ & $1.59(1.32,1.91)$ & $<0.0001$ & $1.73(1.40,2.13)$ & $<0.0001$ \\
\hline \multicolumn{7}{|l|}{ Tertiles } \\
\hline $0-0.2$ & \multicolumn{2}{|c|}{1.0 (ref) } & \multicolumn{2}{|c|}{1.0 (ref) } & \multicolumn{2}{|c|}{1.0 (ref) } \\
\hline $0.21-2.53$ & $1.71(1.01,2.89)$ & 0.0448 & $1.71(1.00,2.91)$ & 0.0496 & $1.57(0.88,2.78)$ & 0.1240 \\
\hline $2.54-16.43$ & $3.65(2.23,5.98)$ & $<0.0001$ & $3.15(1.90,5.20)$ & $<0.0001$ & $2.97(1.70,5.17)$ & 0.0001 \\
\hline$P$ trend & \multicolumn{2}{|c|}{$<0.0001$} & \multicolumn{2}{|c|}{$<0.0001$} & \multicolumn{2}{|c|}{$<0.0001$} \\
\hline \multicolumn{7}{|l|}{ 2-year mortality } \\
\hline Per 1 sd change & $1.88(1.47,2.39)$ & $<0.0001$ & $1.69(1.33,2.16)$ & $<0.0001$ & $1.76(1.35,2.29)$ & $<0.0001$ \\
\hline \multicolumn{7}{|l|}{ Tertiles } \\
\hline $0-0.2$ & \multicolumn{2}{|c|}{1.0 (ref) } & \multicolumn{2}{|c|}{1.0 (ref) } & \multicolumn{2}{|c|}{1.0 (ref) } \\
\hline $0.21-2.53$ & $2.38(1.43,3.97)$ & 0.0009 & $2.53(1.47,4.36)$ & 0.0008 & $2.26(1.26,4.07)$ & 0.0063 \\
\hline $2.54-16.43$ & $4.04(2.44,6.71)$ & $<0.0001$ & $3.40(2.00,5.78)$ & $<0.0001$ & $3.03(1.68,5.47)$ & 0.0002 \\
\hline$P$ trend & \multicolumn{2}{|c|}{$<0.001$} & \multicolumn{2}{|c|}{0.0002} & \multicolumn{2}{|c|}{0.0021} \\
\hline
\end{tabular}

OR: odds ratio; CI: confidence interval. Adjust I model, adjusted for age and gender. Adjust II model, adjust for age, gender, respiratory rate, temperature, $\mathrm{CAD}$, glucose, triglycerides, and surgical grade.

Furthermore, the generalized additive model was generated to measure the independent relationship between CAR and mortality, with adjustment for potential confounders: gender, age, respiratory rate, temperature, CAD, glucose, triglycerides, and surgical grade. The relationship between CAR and mortality was represented by a smooth curve and analyzed by the Chi-square test. $P<0.05$ was considered statistically significant. The data has been analyzed using Empower Stats (http://www.empowerstats.com, X\&Y solutions, Inc. Boston MA) and $\mathrm{R}$ software version 3.6.1 (https://www.r-project.org).

\section{Results}

3.1. Population Characteristics. According to the exclusion criteria, a total of 580 severe patients with AKI were eligible and included in this analysis (Figure 1). Based on CAR tertiles, the patients' population characteristics were described hierarchically and shown in Table 1 . There were 193 patients in the low-CAR group $(\mathrm{CAR}<0.21), 191$ patients in the mid-CAR group (CAR $\geq 0.21,<2.54$ ), and 196 patients in the high-CAR group $(\mathrm{CAR} \geq 2.54)$. This subject included
371 (63.9\%) men and 209 (36.1\%) women. Patients in the high-CAR group had a higher proportion of older people and males and were more likely to suffer from hypertension and coronary heart disease. They also had lower hematocrit, hemoglobin, RBC level, and lower recovery rate. Moreover, these patients had a higher respiratory rate, heart rate, BUN, creatinine, WBC, glucose, triglycerides, TBIL, RDW, BMG, AKI stage, and surgical grade. Finally, they also had longer ICU stays, higher SOFA and APACHE II scores, and higher mortality than those in the low-CAR group.

3.2. CAR Is Associated with Clinical Prognosis. A higher CAR level was associated with higher in-hospital mortality and 2year all-cause mortality in AKI patients admitted to ICU (Table 2). In model I, adjusted for gender and age, and compared with the low-CAR group (CAR $<0.21)$, the high-CAR group (CAR $\geq 16.43$ ) adjusted ORs (95\% CIs) of in-hospital mortality and 2-year all-cause mortality were 3.15 (1.90, $5.20)$ and $3.40(2.00,5.78)$, respectively. In model II, we adjusted for more confounders. We found that CAR remained an independent risk factor for increased inhospital mortality and 2-year all-cause mortality in critically 


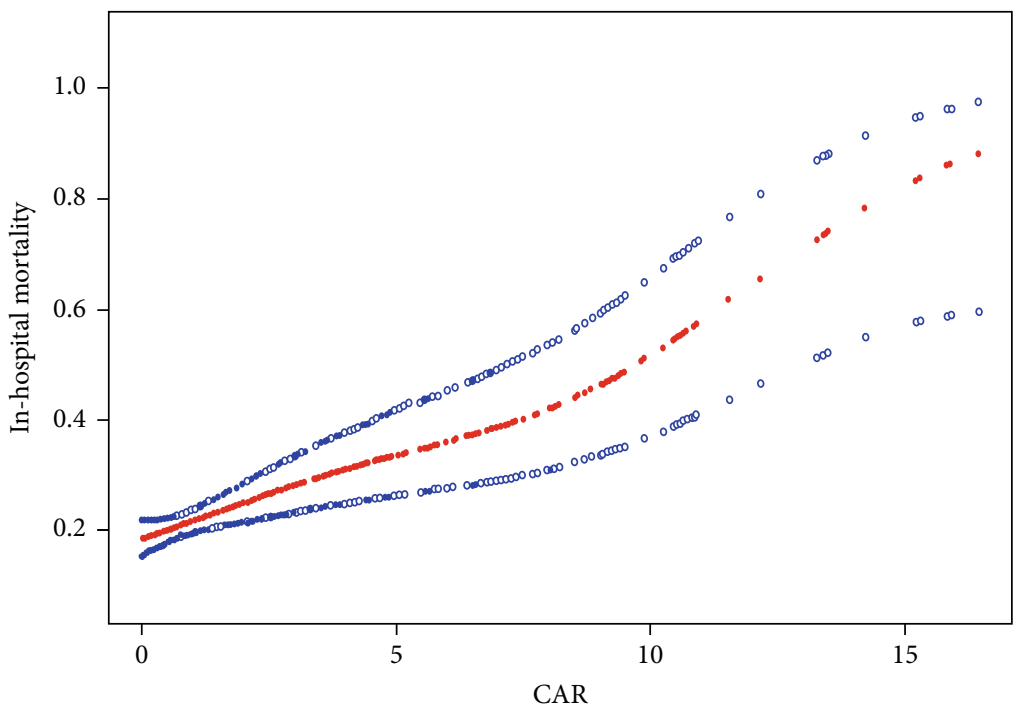

(a)

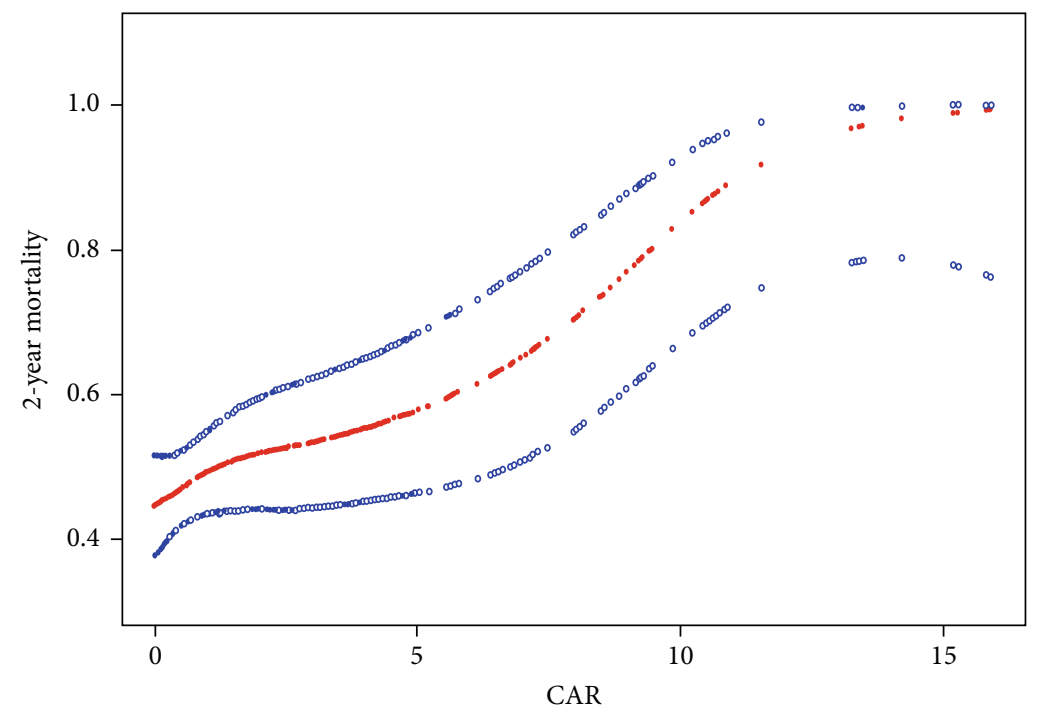

(b)

Figure 2: Association between CAR and in-hospital mortality (a) and 2-year all-cause mortality (b) in critically ill patients with AKI. A linear association between CAR and mortality was found in a generalized additive model (GAM). Solid red line represents the smooth curve fit between variables. Blue bands represent the $95 \%$ of confidence interval from the fit. All adjusted for age, gender, respiratory rate, temperature, $\mathrm{CAD}$, glucose, triglycerides, and surgical grade.

ill patients with AKI (the high-CAR group versus the lowCAR group: adjusted ORs, 95\% CIs, $P$ trends: $2.97,1.70-$ 5.17, <0.0001; 3.03, 1.68-5.47, 0.0002) (Table 2).

After adjusting for confounders, we found a linear relationship between CAR and in-hospital mortality, and the risk of in-hospital mortality of critically ill patients with AKI raised with the increase of CAR $(P<0.0001)$ (Figure 2(a)). Meanwhile, a similar trend was observed in 2-year mortality $(P=0.0043)$ (Figure 2(b)).

3.3. Subgroup Analyses. We performed a stratified analysis to determine that higher CAR levels in each subgroup were consistent with increased in-hospital mortality in patients admitted to ICU with AKI (Table 3). In female patients (OR, 5.4; 95\% CI, 2.1 to 13.9) or who have undergone level
4 surgery (OR, 7.8; 95\% CI, 2.9 to 21.0), the higher the CAR level, the higher risk of the hospital mortality. Similarly, patients with high values of glucose (OR, 6.1; 95\% CI, 2.2 to 17.0 ), TBIL (OR, 6.5; 95\% CI, 2.3 to 18.4), and WBC (OR, 9.6; 95\% CI, 2.2 to 42.9) were at higher risk with increased CAR. The table also shows that in-hospital mortality increases with the increase of CAR regardless of recovery from AKI or not.

\section{Discussion}

In conclusion, we established that a higher level of CAR was associated with a significantly increased risk of in-hospital mortality and 2-year all-cause mortality in patients who developed AKI after admission to the ICU. After adjusting 
TABLE 3: Effect size of CAR on hospital mortality in prespecified and exploratory subgroups in each subgroup.

\begin{tabular}{|c|c|c|c|c|c|}
\hline \multirow{4}{*}{ Gender } & \multicolumn{5}{|c|}{ CRP to albumin ratio (CAR) } \\
\hline & \multirow[t]{2}{*}{$0-0.2$} & \multicolumn{2}{|c|}{$0.21-2.53$} & \multicolumn{2}{|c|}{$2.54-16.43$} \\
\hline & & OR (95\% CIs) & $P$ trend & OR (95\% CIs) & $P$ trend \\
\hline & \multicolumn{5}{|c|}{ Gender } \\
\hline Male & 1.0 (ref) & $1.5(0.8,2.8)$ & 0.223 & $2.9(1.6,5.2)$ & $<0.001$ \\
\hline Female & 1.0 (ref) & $2.2(0.8,6.0)$ & 0.116 & $5.4(2.1,13.9)$ & $<0.001$ \\
\hline \multicolumn{6}{|c|}{ Recovery from AKI } \\
\hline Yes & 1.0 (ref) & $1.3(0.7,2.4)$ & 0.414 & $2.3(1.3,4.3)$ & 0.007 \\
\hline No & 1.0 (ref) & $3.7(1.3,10.7)$ & 0.017 & $8.2(3.0,22.6)$ & $<0.001$ \\
\hline \multicolumn{6}{|l|}{ Age (year) } \\
\hline$<52$ & 1.0 (ref) & $1.7(0.6,4.8)$ & 0.326 & $3.5(1.2,10.2)$ & 0.019 \\
\hline$\geq 53,<66$ & 1.0 (ref) & $2.2(0.8,5.8)$ & 0.110 & $4.6(1.9,10.9)$ & $<0.001$ \\
\hline$\geq 67$ & 1.0 (ref) & $1.4(0.6,3.2)$ & 0.418 & $2.5(1.2,5.3)$ & 0.017 \\
\hline \multicolumn{6}{|c|}{ Respiratory rate (beats/minute) } \\
\hline$<18$ & 1.0 (ref) & $3.2(0.7,14.5)$ & 0.133 & $8.2(2.2,30.9)$ & 0.002 \\
\hline$\geq 18,<20$ & 1.0 (ref) & $2.1(0.7,5.9)$ & 0.158 & $3.4(1.1,10.0)$ & 0.028 \\
\hline$\geq 20$ & 1.0 (ref) & $1.2(0.6,2.4)$ & 0.542 & $2.8(1.5,5.3)$ & 0.001 \\
\hline \multicolumn{6}{|l|}{ BUN (mmol/L) } \\
\hline$<5.6$ & 1.0 (ref) & $1.7(0.7,4.3)$ & 0.230 & $3.9(1.7,9.1)$ & 0.001 \\
\hline$\geq 5.6,<9.4$ & 1.0 (ref) & $1.8(0.7,4.6)$ & 0.206 & $3.9(1.7,9.4)$ & 0.002 \\
\hline$\geq 9.4$ & 1.0 (ref) & $1.4(0.6,3.6)$ & 0.474 & $2.8(1.2,6.9)$ & 0.022 \\
\hline \multicolumn{6}{|c|}{ Creatinine $(\mu \mathrm{mol} / \mathrm{L})$} \\
\hline$<140.09$ & 1.0 (ref) & $1.6(0.6,4.3)$ & 0.388 & $6.1(2.5,15.2)$ & $<0.001$ \\
\hline$\geq 140.09,<207$ & 1.0 (ref) & $2.4(1.0,5.9)$ & 0.055 & $3.9(1.7,9.2)$ & 0.002 \\
\hline$\geq 207$ & 1.0 (ref) & $1.1(0.5,2.6)$ & 0.803 & $2.0(0.9,4.5)$ & 0.109 \\
\hline \multicolumn{6}{|l|}{ Sodium (mmol/L) } \\
\hline$<138$ & 1.0 (ref) & $1.4(0.5,3.8)$ & 0.509 & $3.1(1.1,8.3)$ & 0.028 \\
\hline$\geq 138,<141$ & 1.0 (ref) & $1.8(0.7,4.9)$ & 0.226 & $3.5(1.4,8.9)$ & 0.009 \\
\hline$\geq 141$ & 1.0 (ref) & $1.4(0.6,3.2)$ & 0.478 & $3.4(1.6,7.2)$ & $<0.001$ \\
\hline \multicolumn{6}{|c|}{ Potassium (mmol/L) } \\
\hline$<3.9$ & 1.0 (ref) & $2.4(0.7,8.1)$ & 0.144 & $6.3(2.0,19.7)$ & 0.001 \\
\hline$\geq 3.9,<4.3$ & 1.0 (ref) & $1.6(0.7,3.7)$ & 0.267 & $2.8(1.2,6.4)$ & 0.015 \\
\hline$\geq 4.3$ & 1.0 (ref) & $1.7(0.7,3.9)$ & 0.236 & $3.4(1.6,7.6)$ & 0.002 \\
\hline \multicolumn{6}{|l|}{$\operatorname{RBC}\left(10^{12} / \mathrm{L}\right)$} \\
\hline$<3.71$ & 1.0 (ref) & $2.2(0.7,7.4)$ & 0.180 & $5.2(1.7,16.2)$ & 0.004 \\
\hline$\geq 3.71,<4.5$ & 1.0 (ref) & $1.5(0.6,3.5)$ & 0.360 & $3.5(1.6,8.0)$ & 0.002 \\
\hline$\geq 4.5$ & 1.0 (ref) & $2.3(0.9,5.9)$ & 0.074 & $3.9(1.6,9.6)$ & 0.002 \\
\hline \multicolumn{6}{|l|}{$\mathrm{WBC}\left(10^{9} / \mathrm{L}\right)$} \\
\hline$<6.17$ & 1.0 (ref) & $1.8(0.7,4.2)$ & 0.203 & $4.9(2.1,11.4)$ & $<0.001$ \\
\hline$\geq 6.17,<10.36$ & 1.0 (ref) & $1.9(0.8,4.5)$ & 0.150 & $2.5(1.1,5.8)$ & 0.030 \\
\hline$\geq 10.36$ & 1.0 (ref) & $3.7(0.8,17.3)$ & 0.098 & $9.6(2.2,42.9)$ & 0.003 \\
\hline \multicolumn{6}{|l|}{ Glucose $(\mathrm{mmol} / \mathrm{L})$} \\
\hline$<5.35$ & 1.0 (ref) & $2.0(0.9,4.7)$ & 0.099 & $1.9(0.8,4.4)$ & 0.125 \\
\hline$\geq 5.35,<7.65$ & 1.0 (ref) & $1.1(0.4,2.9)$ & 0.776 & $3.2(1.3,8.0)$ & 0.011 \\
\hline$\geq 7.65$ & 1.0 (ref) & $2.5(0.8,7.5)$ & 0.098 & $6.1(2.2,17.0)$ & $<0.001$ \\
\hline \multicolumn{6}{|l|}{ Hematocrit (\%) } \\
\hline$<33.4$ & 1.0 (ref) & $2.0(0.7,6.1)$ & 0.203 & $4.2(1.5,11.8)$ & 0.007 \\
\hline$\geq 33.4,<40.2$ & 1.0 (ref) & $1.4(0.6,3.3)$ & 0.398 & $3.1(1.3,7.0)$ & 0.008 \\
\hline$\geq 40.2$ & 1.0 (ref) & $2.5(0.9,6.6)$ & 0.067 & $5.6(2.3,13.6)$ & $<0.001$ \\
\hline
\end{tabular}


TABLE 3: Continued.

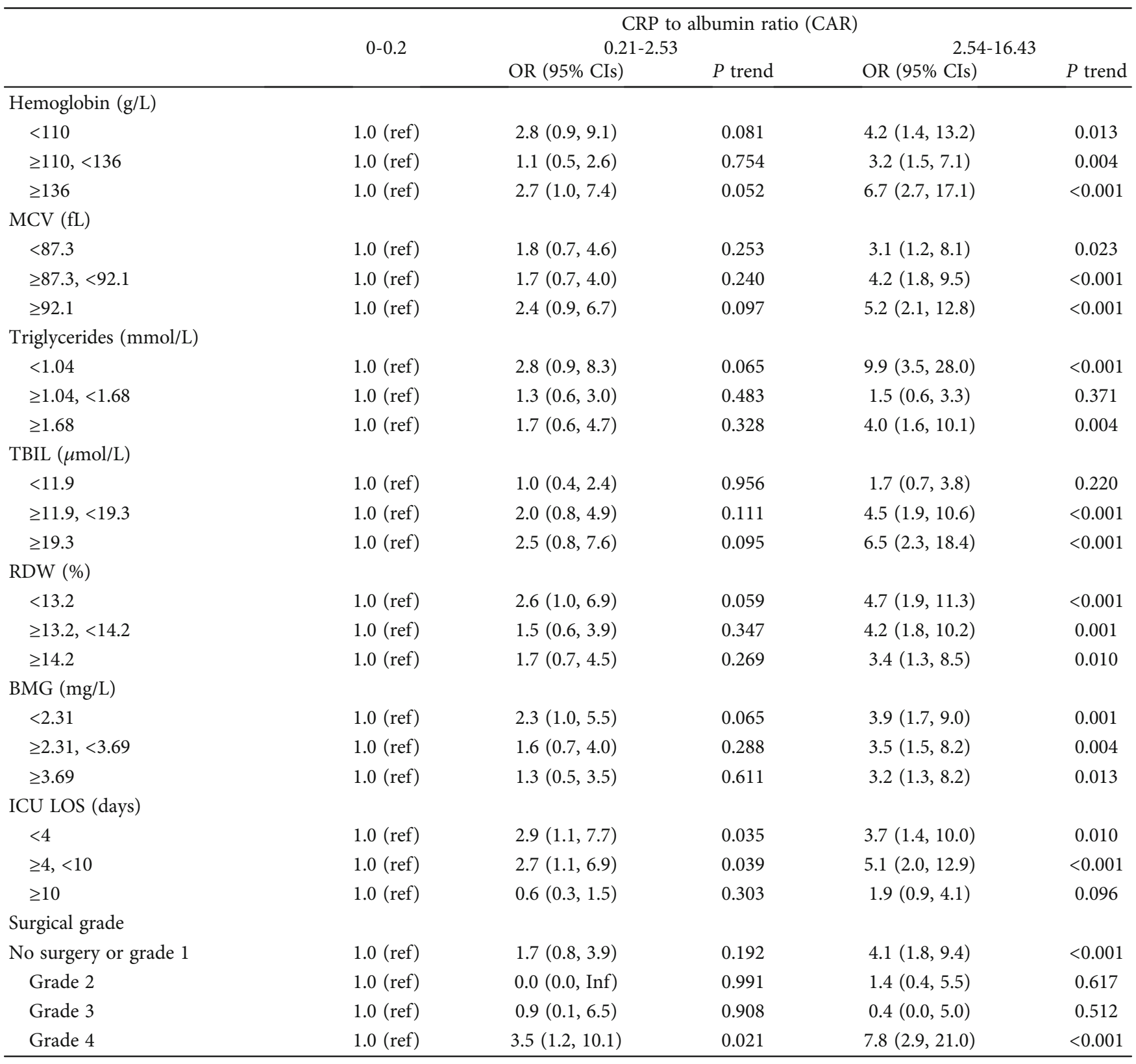

CRP: C-reactive protein; BUN: blood urea nitrogen; RBC: red blood cells; WBC: white blood cell; MCV: mean corpuscular volume; TBIL: total bilirubin; RDW: red cell distribution width; BMG: $\beta 2$-microglobulin; ICU: intensive care unit; LOS: length of stay.

for gender and age, or for more covariates, this significant correlation remains. Both CRP and ALB can be controlled by various clinical treatment methods. The findings of this study may help clinicians to take early treatment measures to reduce the mortality of critically ill patients with AKI.

Although the pathogenesis of AKI remains unclear, previous studies have shown that AKI is associated with high circulating levels of inflammatory mediators [24]. The inflammatory mediators are related to the prognosis of AKI, including CRP, ALB, lymphocytes, tumor necrosis factor receptor I (TNF-R-I), TNF-R-II, neutrophils, platelets, interleukin (IL) 1, IL-6, IL-10, and RDW [25-29]. Among them, CRP, as a significant marker of the inflammatory response, can reduce the expression of endothelial nitric oxide synthase (eNOS) and mRNA stability, promote the expression of endothelial lox-1, stimulate ROS production, and increase endothelial cell apoptosis, and thus promote endothelial dysfunction, which is the main factor of AKI [30]. Tang et al. [31] reported that the role of CRP had been gradually recognized as a factor promoting the occurrence and progression of AKI by preventing the repair and proliferation of damaged renal tubular epithelial cells, increasing inflammatory response, and promoting the fibrosis of damaged renal tissue in recent years. ALB, a protein synthesized in the liver, is generally regarded as a nutritional status marker and has several vital functions, including carrying 
poorly water-soluble molecules, regulating osmotic pressure, antioxidants, and anti-inflammatory effects, which has a protective effect on the kidneys [32, 33]. Hypoalbuminemia makes the body unable to remove toxic substances effectively and leads to decreased vascular volume followed by renal hypoperfusion, and both of them will cause kidney damage [34]. Low ALB serum levels may be associated with systemic inflammatory response syndrome. Several studies have shown that hypoproteinemia can increase the morbidity and mortality of AKI in critically ill patients $[35,36]$.

Therefore, we introduced the concept of CAR, which reflects the combination of inflammation and nutrition. CAR is associated with the prognosis of many diseases, including various cancers (e.g., advanced nonsmall-cell lung cancer and nasopharyngeal carcinoma), inflammatory diseases (e.g., acute pancreatitis and acute severe ulcerative colitis), and cardiovascular diseases [15-18, 37-39]. According to the above mechanism and previous reports, our results were reasonable.

There were some pitfalls and limitations in this study. First, this is an observational, retrospective, single-center study, possibly introducing selection bias. Second, we only calculated the CAR when the patient was admitted to the ICU and did not record the changes in laboratory results during the ICU period, which may cause inaccuracy of the products. Third, attempts have been made to control the study's confounding biases using multivariate analysis techniques, but some unknown confounding factors or information failed to collect. Fourth, we did not have the exact death time of the included cases to do the K-M survival curve. Finally, a retrospective study has some inevitable defects due to its nature; therefore, prospective, multicentered studies should be performed to corroborate these findings.

\section{Conclusions}

We analyzed the clinical data of 580 patients in the electronic medical record system of Shandong Provincial Hospital. Our results show that higher CAR was associated with an increased risk of in-hospital mortality and 2-year all-cause mortality in critically ill patients with AKI. However, further prospective multicenter studies are still needed to confirm our findings.

\section{Data Availability}

The data of this study are all from the electronic medical record system of Shandong Provincial Hospital, and source data can be obtained from the corresponding author Yong Wang if reasonably requested.

\section{Conflicts of Interest}

There is no conflict of interest regarding the publication of this paper.

\section{Acknowledgments}

This work was supported by the Natural Science Foundation of Shandong Province (ZR2020MH307).

\section{References}

[1] R. Bellomo, J. A. Kellum, and C. Ronco, "Acute kidney injury," The Lancet, vol. 380, no. 9843, pp. 756-766, 2012.

[2] J. S. McDonald, R. J. McDonald, E. E. Williamson, D. F. Kallmes, and K. Kashani, "Erratum to post-contrast acute kidney injury in intensive care unit patients: a propensity scoreadjusted study," Intensive Care Medicine, vol. 43, no. 6, pp. 956-956, 2017.

[3] Z. Ricci, D. Cruz, and C. Ronco, "The RIFLE criteria and mortality in acute kidney injury: a systematic review," Kidney international, vol. 73, no. 5, pp. 538-546, 2008.

[4] E. A. Hoste, G. Clermont, A. Kersten et al., "RIFLE criteria for acute kidney injury are associated with hospital mortality in critically ill patients: a cohort analysis," Critical Care, vol. 10, no. 3, p. R73, 2006.

[5] R. L. Mehta, M. T. Pascual, S. Soroko et al., "Spectrum of acute renal failure in the intensive care unit: the PICARD experience," Kidney International, vol. 66, no. 4, pp. 1613-1621, 2004.

[6] H. Schiffl and R. Fischer, "Five-year outcomes of severe acute kidney injury requiring renal replacement therapy," Nephrology Dialysis Transplantation, vol. 23, no. 7, pp. 2235-2241, 2008.

[7] J. H. Westhoff, F. S. Seibert, S. Waldherr et al., "Urinary calprotectin, kidney injury molecule-1, and neutrophil gelatinaseassociated lipocalin for the prediction of adverse outcome in pediatric acute kidney injury," European Journal of Pediatrics, vol. 176, no. 6, pp. 745-755, 2017.

[8] W. R. Zhang and C. R. Parikh, "Biomarkers of acute and chronic kidney disease," Annual Review of Physiology, vol. 81, no. 1, pp. 309-333, 2019.

[9] Z. Zhang, K. M. Ho, and Y. Hong, "Machine learning for the prediction of volume responsiveness in patients with oliguric acute kidney injury in critical care," Critical Care, vol. 23, no. 1, p. 112, 2019.

[10] A. S. Levey and M. T. James, "Acute kidney injury," Annals of Internal Medicine, vol. 167, no. 9, pp. ITC66-ITC80, 2017.

[11] N. Cosentino, S. Genovese, J. Campodonico et al., "High-sensitivity C-reactive protein and acute kidney injury in patients with acute myocardial infarction: a prospective observational study," Journal of Clinical Medicine, vol. 8, no. 12, p. 2192, 2019.

[12] S. N. Murat, A. Kurtul, and M. Yarlioglues, "Impact of serum albumin levels on contrast-induced acute kidney injury in patients with acute coronary syndromes treated with percutaneous coronary intervention," Angiology, vol. 66, no. 8, pp. 732-737, 2015.

[13] D. H. Esper and W. A. Harb, "The cancer cachexia syndrome: a review of metabolic and clinical manifestations," Nutrition in Clinical Practice, vol. 20, no. 4, pp. 369-376, 2005.

[14] Y. Liu, N. Tan, Y. L. Zhou et al., "High-sensitivity C-reactive protein predicts contrast-induced nephropathy after primary percutaneous coronary intervention," Journal of Nephrology, vol. 25, no. 3, pp. 332-340, 2012. 
[15] O. T. Ranzani, F. G. Zampieri, D. N. Forte, L. C. Azevedo, and M. Park, "C-reactive protein/albumin ratio predicts 90-day mortality of septic patients," PLoS One, vol. 8, no. 3, article e59321, 2013.

[16] A. Kinoshita, H. Onoda, N. Imai et al., "The C-reactive protein/albumin ratio, a novel inflammation-based prognostic score, predicts outcomes in patients with Hepatocellular carcinoma," Annals of Surgical Oncology, vol. 22, no. 3, pp. 803-810, 2015.

[17] T. Zhou, J. Zhan, S. Hong et al., "Ratio of C-reactive protein/albumin is an inflammatory prognostic score for predicting overall survival of patients with small-cell lung cancer," Scientific Reports, vol. 5, no. 1, p. 10481, 2015.

[18] M. Wu, J. Guo, L. Guo, and Q. Zuo, "The C-reactive protein/albumin ratio predicts overall survival of patients with advanced pancreatic cancer," Tumor Biology, vol. 37, no. 9, pp. 12525-12533, 2016.

[19] Acute Kidney Injury Network, A. Bagga, A. Bakkaloglu et al., "Improving outcomes from acute kidney injury: report of an initiative," Pediatric Nephrology, vol. 22, no. 10, pp. 16551658, 2007.

[20] N. Pannu, M. James, B. Hemmelgarn, S. Klarenbach, and for the Alberta Kidney Disease Network, "Association between AKI, recovery of renal function, and long-term outcomes after hospital discharge," Clinical Journal of the American Society of Nephrology, vol. 8, no. 2, pp. 194-202, 2013.

[21] J.-L. Vincent, R. Moreno, J. Takala et al., "The SOFA (sepsisrelated organ failure assessment) score to describe organ dysfunction/failure," Intensive Care Medicine, vol. 22, no. 7, pp. 707-710, 1996.

[22] W. A. Knaus, E. A. Draper, D. P. Wagner, and J. E. Zimmerman, "APACHE II: a severity of disease classification system," Critical Care Medicine, vol. 13, no. 10, pp. 818-829, 1985.

[23] G. Maldonado and S. Greenland, "Simulation study of confounder-selection strategies," American Journal of Epidemiology, vol. 138, no. 11, pp. 923-936, 1993.

[24] Y. Karabağ, M. Çağdaş, I. Rencuzogullari et al., "The Creactive protein to albumin ratio predicts acute kidney injury in patients with ST-segment elevation myocardial infarction undergoing primary percutaneous coronary intervention," Heart, Lung \& Circulation, vol. 28, no. 11, pp. 1638-1645, 2018.

[25] Z. Forhecz, T. Gombos, G. Borgulya, Z. Pozsonyi, Z. Prohaszka, and L. Janoskuti, "Red cell distribution width in heart failure: prediction of clinical events and relationship with markers of ineffective erythropoiesis, inflammation, renal function, and nutritional state," American Heart Journal, vol. 158, no. 4, pp. 659-666, 2009.

[26] D. Payen, A. C. Lukaszewicz, M. Legrand et al., "A multicentre study of acute kidney injury in severe sepsis and septic shock: association with inflammatory phenotype and HLA genotype," PloS One, vol. 7, no. 6, article e35838, 2012.

[27] M. Araujo, S. Q. Doi, C. E. Palant, E. S. Nylen, and K. L. Becker, "Procalcitonin induced cytotoxicity and apoptosis in mesangial cells: implications for septic renal injury," Inflammation Research Official Journal of the European Histamine Research Society, vol. 62, no. 10, pp. 887-894, 2013.

[28] A. Rudiger and M. Singer, "The heart in sepsis: from basic mechanisms to clinical management," Current Vascular Pharmacology, vol. 11, no. 2, pp. 187-195, 2013.

[29] O. Takasu, J. P. Gaut, E. Watanabe et al., "Mechanisms of cardiac and renal dysfunction in patients dying of sepsis," Amer- ican Journal of Respiratory and Critical Care Medicine, vol. 187, no. 5, pp. 509-517, 2013.

[30] H. Fujii, S. H. Li, P. E. Szmitko, P. W. Fedak, and S. Verma, "Creactive protein alters antioxidant defenses and promotes apoptosis in endothelial progenitor cells," Arterioscler. Thromb. Vasc. Biol, vol. 26, no. 11, pp. 2476-2482, 2006.

[31] Y. Tang, S.-K. Mak, A. P. Xu, and H.-Y. Lan, "Role of Creactive protein in the pathogenesis of acute kidney injury," Nephrology, vol. 23, Supplement 4, pp. 50-52, 2018.

[32] J. L. Vincent, "Relevance of albumin in modern critical care medicine," Best Practice \& Research Clinical Anaesthesiology, vol. 23, no. 2, pp. 183-191, 2009.

[33] M. Y. Yu, S. W. Lee, S. H. Baek et al., "Hypoalbuminemia at admission predicts the development of acute kidney injury in hospitalized patients: a retrospective cohort study," PLoS One, vol. 12, no. 7, article e0180750, 2017.

[34] A. M. Contreras, M. Ramirez, L. Cueva, S. Alvarez, R. de Loza, and G. Gamba, "Low serum albumin and the increased risk of amikacin nephrotoxicity," Revista de Investigacion Clinica; Organo del Hospital de Enfermedades de la Nutricion, vol. 46, no. 1, pp. 37-43, 1994.

[35] C. J. Wiedermann, W. Wiedermann, and M. Joannidis, "Hypoalbuminemia and acute kidney injury: a meta-analysis of observational clinical studies," Intensive Care Medicine, vol. 36, no. 10, pp. 1657-1665, 2010.

[36] C. Tongprayoon, W. Cheungpasitporn, M. A. Mao, A. Sakhuja, and K. Kashani, "U-shape association of serum albumin level and acute kidney injury risk in hospitalized patients," PloS One, vol. 13, no. 6, article e0199153, 2018.

[37] I. Rencuzogullari, Y. Karabag, M. Cagdas et al., “Avaliaçao preprocedimento da relaçao entre a proteina C-reativa e a albumina na reestenose de stents em doentes com enfarte do mioc ardio com elevaçao do segmento ST," Revista Portuguesa de Cardiologia, vol. 38, no. 4, pp. 269-277, 2019.

[38] M. Kaplan, I. Ates, M. Y. Akpinar et al., "Predictive value of Creactive protein/albumin ratio in acute pancreatitis," Hepatobiliary \& Pancreatic Diseases International, vol. 16, no. 4, pp. 424-430, 2017.

[39] D. J. Gibson, K. Hartery, J. Doherty et al., “CRP/albumin ratio: an early predictor of steroid responsiveness in acute severe ulcerative colitis," Journal of Clinical Gastroenterology, vol. 52, no. 6, pp. e48-e52, 2018. 